\title{
Massage Therapy for Lyme Disease Symptoms: a Prospective Case Study
}

\author{
Meghan J. Thomason, BA, MT, Christopher A. Moyer, PhD \\ Department of Psychology, University of Wisconsin-Stout, Menomonie, WI, USA
}

\begin{abstract}
Introduction: To study the effects of massage therapy (MT) on Lyme disease (LD) symptoms and affect.

Methods: A 21-year-old female college student previously diagnosed with $L D$ was recruited for a prospective case study that incorporated alternating periods of treatment and nontreatment across 65 days. Her self-reported symptoms of pain, fatigue, and impairment of concentration were assessed by means of a daily diary with corresponding visual analog scales. Immediate effects of MT on affect were assessed by completion of the Positive and Negative Affect Scales before and after each treatment session.

Results: LD symptoms decreased during treatment periods and increased during nontreatment periods. Positive affect was increased at every MT session.

Conclusions: MT is a promising treatment for the symptoms pain, fatigue, and impaired concentration associated with $L D$. In addition, MT reliably increased positive affect. Massage therapists should consider using light-to-medium pressure MT for treatment of persons who present with a similar pattern of $L D$ symptoms, and further research with this population is warranted.
\end{abstract}

KEYWORDS: Lyme disease, rheumatoid arthritis, affect, massage therapy, prospective case study, pain, concentration, fatigue, arthritis, quasi-experiment

\section{INTRODUCTION}

Lyme disease (LD) is a chronic illness caused by a bacterium carried by ticks and transmitted by their bite. Prevalence varies by region; in Wisconsin, where the present case study was conducted, approximately 26 of every 100,000 people were diagnosed with LD during 2008. Individuals who work outdoors or engage in high amounts of outdoor recreation are more likely to be infected with LD than individuals who spend most of their time indoors ${ }^{(1)}$. The initial symptom is commonly the appearance of a red, targetlike rash caused by the person's immune system response to the infection. However, this rash does not necessarily appear at the site of the tick bite, and in some cases it does not appear at all. Subsequently, flu-like symptoms such as achiness, chills, swollen glands, and headache are commonly experienced. Eventually, deep nerve and joint pain similar to that experienced with rheumatoid arthritis may occur ${ }^{(2)}$.

The bacteria that cause LD predominantly migrate to the nervous system and joint tissues, which protects them from their host's immune system, and only rarely do they attack vital organs. However, the brain, a nervous system structure, is vulnerable to attack by the LD bacteria, and this can lead to impairments such as excessive fatigue, an inability to concentrate, or loss of short-term memory ${ }^{(2)}$. Inflammation of the facial nerves can also occur, resulting in symptoms similar to Bell's palsy ${ }^{(3)}$. In rare cases the heart, liver, eyes, or other organs are affected. Complications of advanced LD, such as bradycardia or liver failure, can be fatal; however, antibiotic treatment is usually effective, and death from LD is rare ${ }^{(2)}$.

Diagnosis of LD by physiological means, such as a blood test, is imprecise, as the LD bacteria do not occur in high concentrations at any specific anatomical site to permit reliable detection. False negatives are common in LD tests, and often several tests are conducted before LD antibodies are found. In addition, because the predominant symptoms are similar to those of rheumatoid arthritis, people suffering from LD are commonly misdiagnosed with that condition $^{(2)}$. Once LD is diagnosed, treatment with antibiotics, such as doxycycline, is standard ${ }^{(4)}$. Treatment duration can last from several weeks to several years, depending on the severity of the infection, and the side effects experienced during antibiotic treatment include nausea, vomiting, and photosensitivity ${ }^{(5)}$. In addition, the joint pain caused by LD often persists after treatment has concluded, and additional antibiotic treatment does not appear to reduce the lingering symptoms of $\mathrm{LD}^{(6)}$, which may resolve spontaneously or persist indefinitely ${ }^{(2)}$.

Expert opinion suggests that massage therapy (MT) could benefit a person with $\mathrm{LD}^{(7)}$, though presently there appears to be no direct evidence to support this. However, MT is an effective treatment for rheumatoid arthritis ${ }^{(8,9,10)}$, a condition with a similar presentation and for which joint pain is also 
a key symptom, and is also a promising treatment for anxiety ${ }^{(11)}$ and headache ${ }^{(12)}$, both of which may occur with LD. Consequently, it is logical that MT could be an effective treatment for LD symptoms and that investigation is warranted.

\section{METHODS}

\section{Research Design}

Approval from University of Wisconsin-Stout's Institutional Review Board was obtained prior to study commencement. Because this was a prospective case study, the inclusion of treatment and withdrawal periods according to a predetermined schedule was possible. This valuable study feature permits comparison of treatment and nontreatment periods, which has the potential to illustrate treatment effectiveness more clearly than study designs without nontreatment periods ${ }^{(13)}$. The study consisted of five phases. Phase I began with an intake interview to assess the participant's symptoms, after which a nine-day period of nontreatment began. During Phase II, six MT sessions were administered across a three-week period. Phase III was a one-week nontreatment period during which MT was withdrawn. During Phase IV MT resumed as in Phase II. The study concluded with Phase V, a nine-day nontreatment period. The study's total duration was 65 days, and concluded with a qualitative interview that provided an opportunity for the participant to share her experience and opinions with the researchers.

\section{Profile of Participant}

The participant was a 21-year-old female college student with LD recruited for study participation by her college professor, the second author (C.A.M.) of the present study. An intake interview revealed that the participant's physician believed the participant had contracted LD when she was nine or ten years old, but the specific time of infection was unknown. The first symptom the participant could recall was numbness in her toes beginning around age 12. As the condition progressed, she began to experience stiffness in her joints and sharp, throbbing pain in numerous anatomical sites that significantly affected her daily life by age 15 . By age 16 , pain had spread to all her joints and to her muscles, which caused frequent school absences. It was not until age 19 that she received a diagnosis of $\mathrm{LD}$, ten years after the estimated time of infection. The course of her illness and symptom pattern appeared to be typical of individuals with undiagnosed LD of similar duration ${ }^{(1)}$.

Diagnosis led to treatment with antibiotics, which lasted for two years. During this period the participant experienced nausea, vomiting, and photosensitivity that were attributed to the antibiotic treatment. This course of treatment, which reduced but did not eliminate the participant's LD symptoms, ended several months before the present case study commenced. Consequently, the participant was eager to explore new treatment options that might reduce her residual symptoms. She had previously explored chiropractic and craniosacral therapies for pain relief, but believed they had only yielded minor benefits. Though she was uncertain how she would respond to MT, she was interested in trying it and hoped it would provide some pain relief.

\section{Setting and Equipment}

Interviews and MT sessions were conducted in University of Wisconsin-Stout's Psychology Department research laboratories. A room configured for MT-related research, including a professional massage table (Stronglite; Salt Lake City, UT), was used for treatment sessions, while intake and termination interviews were conducted in an adjacent office. Massage cream (PurePro ProTouch Massage Cream; Greenfield, MA) was used to facilitate treatment and, because the room was air-conditioned to $\sim 68^{\circ}$ Fahrenheit, a table warmer (ElectroWarmth Products, LLC, Danville, OH), was used for all sessions to further ensure the participant's comfort. To ensure consistency, the same two musical selections ${ }^{(14,15)}$ were played as accompaniment at each MT session.

\section{Therapist}

The therapist and first author of the present study (M.J.T.) graduated from Wisconsin Indianhead Technical College in 2007 with a Certificate in Therapeutic Massage. She completed a Bachelor's degree in psychology and is currently completing a Master's degree in applied psychology, with dual emphases in health promotion and research evaluation, both from University of Wisconsin-Stout. She emphasizes deep tissue massage techniques, but adapts this approach to meet individual preferences and clinical circumstances.

\section{Treatment}

A standard full-body MT protocol was used as a starting point for treatment. The participant undressed to her level of comfort, and was draped for each session, such that only the anatomical site being worked on at a particular time was exposed. MT sessions began with the participant prone on the massage table. Massage was then applied to the back and neck for 15 minutes, followed by the back of each leg for 5 minutes each. The participant then turned supine, and massage was applied to the front of each leg, and the feet, arms, and hands for a total of 20 minutes. The session concluded with approximately 5 minutes of massage to the head, jaw, neck, and shoulders. Sessions were approximately 50 minutes in length. Prior 
to each MT session, the participant was asked about aches and pains that were troubling on that particular day, such that alterations to the treatment protocol could be made to accommodate her specific needs. Additional focus to the jaw, scalp, and upper back were requested at nearly every treatment session.

Though some research suggests that moderate pressure may be necessary for beneficial MT effects ${ }^{(16)}$, the participant had previously experienced a deep-tissue massage and believed that the associated pressure increased, rather than reduced, her pain. Therefore, the predominant massage technique applied across MT sessions in this study was light effleurage corresponding to Walton's ${ }^{(17)}$ Levels Two ("heavy lotioning" including "slight movement of superficial adipose tissue and muscle") and Three ("medium pressure" including "some movement of medium layers of adipose tissue, muscles, and blood vessels"), consistent with the participant's preference for minimal pressure.

\section{Assessments}

\section{Daily diary assessment of symptoms}

A daily diary, which the participant would complete at the end of each day during the study, was developed with her input. She identified pain, fatigue, and impairment of concentration as the most salient LD symptoms; therefore, each day's entry included a separate 13-centimeter visual analogue scale (VAS) for pain level, fatigue, and impairment of concentration experienced that day. The participant intersected the VAS to indicate the severity of each symptom that day, ranging from none to maximum. Additional space was provided for the participant to provide any qualitative information that she wished to add.

\section{Affect assessment at each treatment}

Immediately before and after each massage session, the participant completed the Positive and Negative Affect Scale (PANAS) ${ }^{(18)}$. This enabled an examination of the immediate effect of MT on the participant's emotional state. Each of the 20 PANAS items consists of an adjective followed by a five-level rating scale that ranges from "not at all" (corresponding to a score of 1 for that item) to "extremely" (corresponding to a score of 5 for that item). Ten items (e.g., excited, interested) assess the respondent's positive affect, the degree to which the person feels enthusiastic, active, and alert; the other ten items (e.g., scared, irritable) assess negative affect, the degree to which the person feels distressed or otherwise experiences aversive mood states. Possible scores for each subscale range from 10 to 50. The PANAS has been widely used and is extensively validated ${ }^{(19,20)}$.

\section{Analysis of quantitative data}

Because this study includes data from a single participant only, it was expected that power for any inferential statistical tests would be low; therefore, a visual approach that made use of charting the data was emphasized. Nevertheless, some descriptive and inferential statistics were computed. For example, the change in PANAS positive affect subscale scores was examined by performing a paired-samples t-test that compared pre-MT and post-MT scores.

Daily diary VAS data were quantified by using a standard ruler to measure where the participant had intersected each $13 \mathrm{~cm}$ VAS line to indicate her level of a specific LD symptom for each day. Each of the two researchers conducted this process independently, so that measurement and coding accuracy could be checked. Metric units were then mathematically transformed to a 0 to 100 scale. Correlation coefficients for the three LD symptoms were examined to determine if the symptoms could meaningfully be combined into a composite LD symptom score, after which symptom scores were charted to permit visual examination. Finally, a web-based statistical tool (http://faculty. vassar.edu/lowry/corr stats.html) was used to calculate regression lines and associated statistics for each of the five study phases so that trends could be more carefully visualized and quantified.

\section{RESULTS}

The case study was completed over a period of 65 days, during which no unexpected circumstances or events interfered with the planned collection of data or delivery of MT. The participant was able to complete the daily diary of LD symptoms each day, and attended all 12 of the twice-weekly MT sessions.

\section{Quantitative}

\section{Lyme disease symptoms}

Prior to any analysis, the researchers' independent measurements of the participant's VAS data for the LD symptoms of pain, fatigue, and impairment in concentration were checked for interrater reliability; all three were highly reliable (rs $>0.99$ ). Further, the three LD symptom variables were all strongly correlated across the 65-day study period (concentration and fatigue, $r(63)=.81$; pain and fatigue $r(63)=.78$; and concentration and pain, $\mathrm{r}(63)=.72)$. Given the consistently high correlations among the symptoms, the decision was made to average them to yield a LD composite symptom score.

Across the entire study, the participant's composite symptom score varied considerably, ranging from 18.08 to 90.36 . Regression slopes calculated and graphed for each of the five study phases suggest that some of this variation was systematically associated with treatment. During Phase I, a nontreatment period, the participant's composite symptom score increased an average of 0.38 points per day. During treatment in Phase II, the composite symptom score decreased an 
average of 0.69 points per day. When treatment was withdrawn in Phase III, the composite symptom score increased an average of 0.74 points per day. When treatment was reintroduced in Phase IV, the composite symptom score decreased an average of 1.54 points per day. Finally, when treatment was withdrawn again in Phase $\mathrm{V}$, the composite symptom score increased an average of 2.59 points per day. In sum, symptoms tended to decrease during MT treatment periods, and to increase during nontreatment periods. In addition, a more rapid rate of improvement and the most consistent period of reduced LD symptoms occurred during the second phase of treatment (Phase IV), which suggests that MT was more effective during the second treatment phase; these trends, along with the composite symptom scores for each day, are presented in Figure 1.

\section{Affect}

A meaningful examination of the effect of MT on negative affect was not possible in the present study, as the participant's pretreatment level of negative affect neared the minimum possible subscale score (of $10)$ at each MT session ( mean $=11.67, \mathrm{SD}=2.61$ ). Predictably, post-treatment levels of negative affect were in the same range (mean $=11.41, \mathrm{SD}=1.24)$. By contrast, the participant's pretreatment levels of positive affect $($ mean $=32.58, \mathrm{SD}=3.75)$ were closer to the midrange of the PANAS subscale, and increased significantly in response to massage sessions (mean $=$ $37.41, \mathrm{SD}=3.90$; paired samples $\mathrm{t}(11)=11.42, \mathrm{r}=$ $.93, p<.001)$. In addition, positive affect appears to increase across the study periods during which it was assessed. These data are presented graphically in Figure 2.

\section{Qualitative}

The areas that the participant indicated as being painful or troublesome were consistent through the data collection. These were most often the upper back,

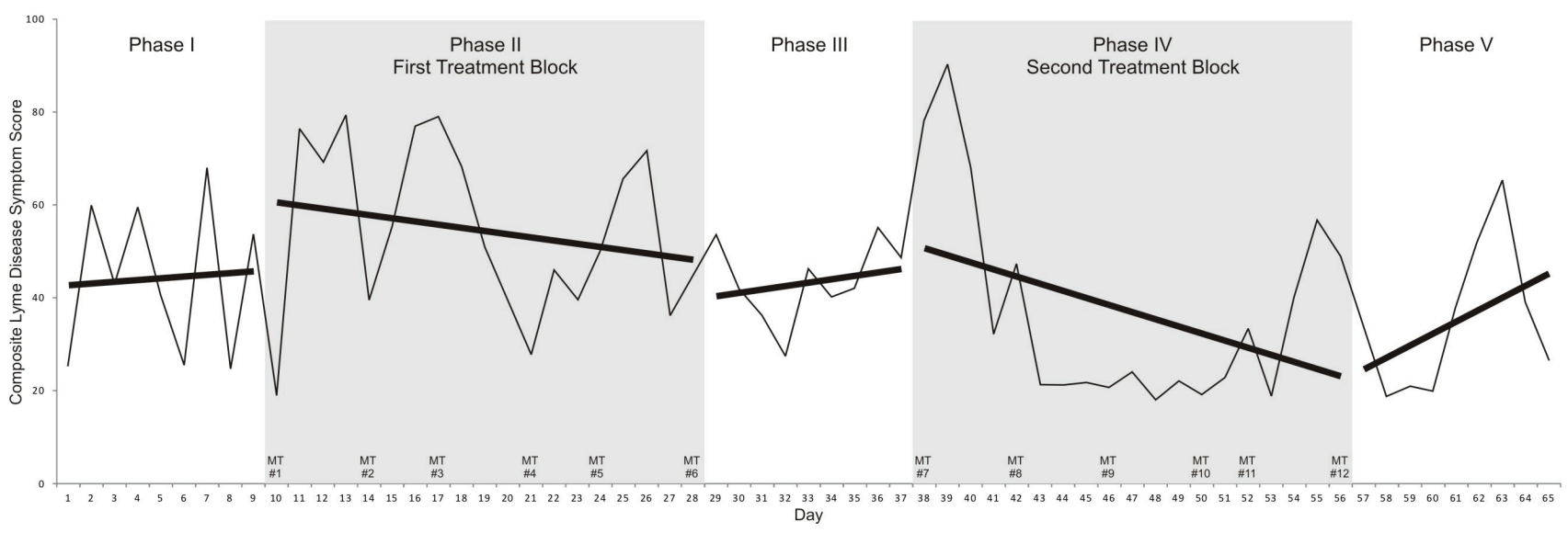

FIGURE 1. LD composite symptom scores by day, and the associated regression lines for each of five study phases.

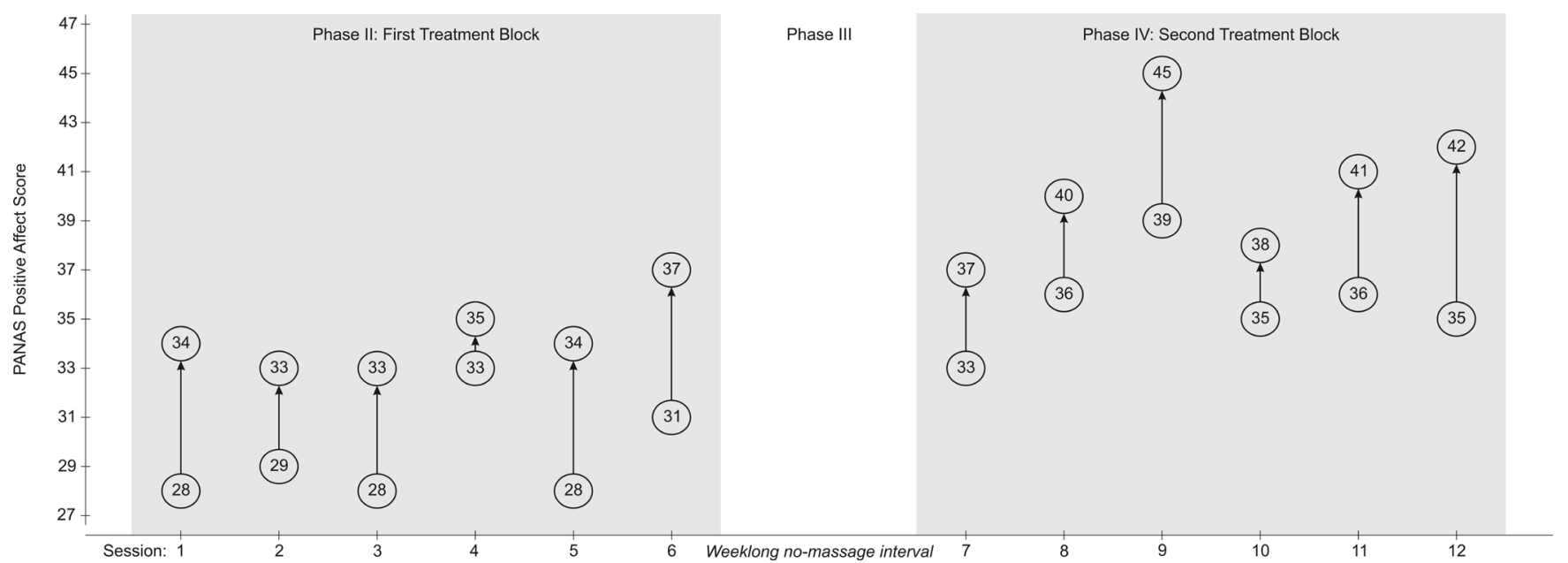

FIGURE 2. PANAS positive affect scores before and after MT sessions. 
the jaw, and the ankles. The participant's muscle tension, as perceived by the therapist, varied little across MT sessions, and the rhomboid and trapezius muscles were consistently firm to the touch. Consistent with her quantitative daily diary assessments, the participant's pretreatment reports of her recent symptoms indicated that they were highly variable. She reported that her level of pain varied depending on her activity level during the previous day and the amount of sleep she had that night.

During the interview conducted at the conclusion of the study, the participant reported that she would feel some pain and tenderness immediately following MT sessions, but noted that she would feel better after a few hours and that the duration of feeling better "around her joints" would last for two to three days following massage sessions. She also indicated that she found scalp massage to be especially beneficial, and that it gave her a "tingly, almost numbing sensation" She found this to be pleasant and reported that she would have liked more treatment time devoted to this.

\section{DISCUSSION \& CONCLUSIONS}

This case study presents evidence that MT can be an effective treatment for the symptoms of LD. The participant's LD symptoms decreased during treatment periods, and her positive affect increased at every treatment session. Though she experienced some mild pain and tenderness immediately following sessions, she reported that these dissipated in a matter of hours, that she enjoyed massage therapy, and that it helped her to feel better for several days after a session. She reported that she was always eager to attend the MT sessions, which was reflected in her timely attendance of all scheduled appointments.

Though there is considerable variability in the LD composite symptom scores, their pattern suggests some interesting trends. LD symptom reduction in response to the first several sessions of MT appears to be minimal, and then increases with the accumulation of sessions. This suggests that there may be an adjustment period for someone with LD during which they get used to receiving MT, or that there may be an adaptive process during which the therapist is learning the best way to massage a particular individual. Future research, including studies that make use of multiple participants and experimental methodology, should more carefully examine such dosage and experience effects.

An unexpected finding was that the participant found scalp massage particularly pleasant and beneficial. She speculated that, given enough time, the scalp massage and the sensations it produced could cause her to sleep, an effect that could be desirable for a person with LD when insomnia is a symptom. She also indicated that she slept better when she was having less pain; therefore, if MT was reducing her pain, it may also have been improving her sleep. Precise assessment of the participant's duration and quality of sleep by means of an actigraph or electroencephalography was not practical in the current case study, but subsequent studies could use such methods to examine if MT improves sleep by means of pain reduction or other mechanisms.

The inclusion of planned treatment and withdrawal periods in the present case study was illuminating and helped us determine with some confidence that massage therapy was responsible for reduction of the participant's Lyme disease symptoms. We recommend this approach to other massage therapists who may undertake prospective case studies. Except in cases where the withdrawal of a highly effective treatment would be unethical, the use of planned treatment and withdrawal periods strengthens the potential of case study methodology to uncover treatment effectiveness.

Nevertheless, case studies, including the present study, have inherent limitations. For example, it cannot be known if the effects reported here are unique to this particular participant, or if they would be observed in most persons with a similar history of LD. Larger and more scientifically rigorous studies could more thoroughly examine the effect of MT on LD symptoms. Further, though the planned inclusion of treatment and withdrawal periods aided us in examining treatment effectiveness, a more rigorous study design that employs a control group would be a more definitive test of treatment effects. Finally, the participant completed her assessment of daily LD symptoms in the evening, which was the time of day she tended to experience the highest levels of pain. As a result, the data for this and related symptoms may have been biased by this form of retrospective self-report, and future studies might address this by assessing pain by other means or according to a different schedule.

Given that MT was experienced as both beneficial and pleasant in the current case study, and that the data provide objective evidence that it improved affect and reduced LD symptoms, massage therapists should consider using light-to-medium pressure MT for treatment of persons who present with a similar pattern of LD symptoms. Further research with this population is also warranted.

\section{CONFLICT OF INTEREST NOTIFICATION}

The authors declare there are no conflicts of interest.

\section{COPYRIGHT}

Published under the CreativeCommons AttributionNonCommercial-NoDerivs 3.0 License. 


\section{REFERENCES}

1. Lyme Disease. Centers for Disease Control and Prevention. 2011. http://www.cdc.gov/lyme/ Retrieved 27 June 2011.

2. Barbour A. Lyme Disease: The Cause, the Cure, the Controversy. Baltimore, MD: John Hopkins University Press; 1996.

3. Markby D. Lyme Disease facial palsy: differentiation from Bell's Palsy. Br Med J. 1989;229(6699):605-606.

4. Dillon R, O'Connell S, Wright S. Lyme Disease in the UK: clinical and laboratory features and response to treatment. Clin Med. 2010;10(5):454-457.

5. Bryant SG, Fisher S, Kluge RM. Increased frequency of doxycycline side effects. Pharmacotherapy. 1987;7(4):125-129.

6. Klempner M, Hu L, Evans J, et al. Two controlled trials of antibiotic treatment in patients with persistent symptoms and a history of Lyme Disease. N Eng J Med. 2001;345(2):85-92.

7. Werner R. Massage Therapist's Guide to Pathology. Baltimore, MD: Lippincott, Williams, \& Wilkins; 2003.

8. Bieder S, Moyer CA. Randomized controlled trials of pediatric massage: a review. Evidence Based Complimentary and Alternative Medicine. 2007;4(1):23-34.

9. Field T, Hernandez-Reif M, Seligman S, et al. Juvenile rheumatoid arthritis: benefits from massage therapy. J Pediatr Psychol. 1997;22(5):607-617.

10. Unsal A, Gozum S. Use of complementary and alternative medicine by patients with arthritis. J Clin Nursing. 2001;45(4):1129-1138.

11. Moyer CA. Anxiety and depression: overview, p.151-164. In: Dryden T, Moyer CA, editors. Massage Therapy: Integrating Research and Practice. Champaign, IL: Human Kinetics; 2012.
12. Moraska A. Headache types, p.115-127. In: Dryden T, Moyer CA, editors. Massage Therapy: Integrating Research and Practice. Champaign, IL: Human Kinetics; 2012.

13. Cozby PC, Bates SC. Methods in Behavioral Research, 11th edition. New York: McGraw-Hill; 2012.

14. Brewer S. Portraits [CD]. New York: Narada Publishing; 1990.

15. Lanz D. Cristifori's Dream [CD]. New York: Narada Publishing; 1999.

16. Field T, Diego M, Hernandez-Reif M. Moderate pressure is essential for massage therapy effects. Int $J$ Neurosci. 2010;120(5):381-385.

17. Walton T. Medical Conditions and Massage Therapy: a Decision Tree Approach. Baltimore, MD: Lippincott Williams and Wilkins; 2010.

18. Watson D, Clark LA, Tellegen A. Development and validation of brief measures of positive and negative affect: The PANAS scales. J Per Soc Psychol. 1988;54(6):1063-1070.

19. Crawford JR, Henry JD. The positive and negative affect schedule (PANAS): construct validity, measurement properties and normative data in a large non-clinical sample. Br J Clin Psychol. 2004;43(Pt 3):245-265.

20. Conner TS, Barrett LF. Trends in ambulatory self-report: the role of momentary experience in psychosomatic medicine. Psychosom Med. 2012;74(4):327-337.

Corresponding author: Christopher A. Moyer, $\mathrm{PhD}$ E-mail: christopher.a.moyer@gmail.com 